ARTICLE

\title{
Estimation of Sample Reactivity Worth with Differential Operator Sampling Method
}

\author{
Yasunobu NAGAYA* and Takamasa MORI \\ Japan Atomic Energy Agency, Tokai-mura, Naka-gun, Ibaraki-ken, 319-1195, Japan
}

\begin{abstract}
Applicability of the Taylor series approach with the arbitrary-order differential operator sampling (DOS) method is examined for the calculation of sample reactivity worth. The DOS method is extended to obtain the differential coefficients of the effective multiplication factor and the perturbed source effect up to the arbitrary order. The methodology is implemented into a continuous-energy Monte Carlo code MVP to perform benchmark calculations. It is found that the second-order Taylor series approach gives an enough accurate result for simple fast systems of Godiva and Jezebel. A discrepancy of $\sim 10 \%$ is, on the other hand, observed for the Np-237 sample worth calculation for the tank-type critical assembly TCA even with the fifth-order Taylor series approach. The perturbed source effect has a significant contribution for the calculation of sample reactivity worth; it must be estimated for all the cases. Alternative approaches such as the Padé approximation, the midpoint and interpoint methods are also examined for the TCA sample worth problem. They give similar results as the ordinary higher-order Taylor series approach.
\end{abstract}

\section{KEYWORDS: Monte Carlo, perturbation, sample reactivity worth, differential operator sampling, perturbed source effect}

\section{Introduction}

Nuclear reactor analysis requires calculations of reactivity worth such as control rod worth, void reactivity worth, sample reactivity worth, etc. It is, however, difficult to perform such calculations with the Monte Carlo method if the worth is small. In such cases, it might be appropriate to use Monte Carlo perturbation methods: correlated sampling and differential operator sampling (DOS) methods. Applicability of these methods to reactivity changes has been extended by taking the perturbed source effect (PSE) into account. ${ }^{1,2)}$ One, however, must note that the methods are applicable only for small perturbation such as small fractional density change; the variance diverges for large perturbation in the correlated sampling method and the higher-order effect is neglected in the DOS method.

In the present work, applicability of the Taylor series approach with the DOS method is examined for the calculation of sample reactivity worth. The DOS method is extended to obtain the differential coefficients of the effective multiplication factor up to an arbitrary order. The extension has been already proposed by Morillon ${ }^{3)}$ but we present a formulation to calculate the contributions from the PSE explicitly. The normalization condition is also derived for the higher-order differential coefficient of the effective multiplication factor.

The extended DOS method is implemented into the MVP $\operatorname{code}^{4)}$ to perform benchmark calculations. The benchmark systems are Godiva, Jezebel and TCA. ${ }^{5)}$ The first two are simple fast systems and the last is a complicated thermal system. We do not focus on the comparison between the calculated

*Corresponding author, E-mail:nagaya.yasunobu@jaea.go.jp

(C) 2011 Atomic Energy Society of Japan, All Rights Reserved. and measured values though measurements of sample reactivity worth were performed at Jezebel and TCA. The comparison is performed with the reference values obtained by two independent Monte Carlo calculations to investigate the applicability of the Taylor series approach.

\section{Methodology}

The change in the effective multiplication factor $k_{i}$ of the $i$-th generation can be expressed with the Taylor series expansion as

$$
\begin{aligned}
\Delta k_{i} & =\frac{\partial k_{i}}{\partial a} \Delta a+\frac{1}{2} \frac{\partial^{2} k_{i}}{\partial a^{2}}(\Delta a)^{2}+\cdots \\
& +\frac{1}{n !} \frac{\partial^{n} k_{i}}{\partial a^{n}}(\Delta a)^{n}+\cdots
\end{aligned}
$$

where $a$ is a perturbation parameter such as a material density, etc. Each differential coefficients at a given state can be estimated with the DOS method.

\section{First-Order Differential Coefficient}

According to the DOS formulation by Nagaya and Mori, ${ }^{1)}$ the first-order differential coefficient of $k_{i}$ can be written as follows:

$$
\begin{aligned}
\frac{\partial k_{i}}{\partial a} & =\frac{1}{\int d P S_{f, i}(P)} \sum_{m=1}^{\infty} \int d P_{m} \cdots \int d P_{1} \int d \boldsymbol{r}_{0} \\
& \times W_{f}^{(1)}\left(P_{m}, \cdots, P_{1}, \boldsymbol{r}_{0}\right) F\left(P_{m}, \cdots, P_{1}, \boldsymbol{r}_{0}\right)
\end{aligned}
$$




$$
\begin{aligned}
& F\left(P_{m}, \cdots, P_{1}, \boldsymbol{r}_{0}\right)= \\
& \quad \frac{\nu \Sigma_{f}\left(P_{m}\right)}{\Sigma_{t}\left(P_{m}\right)} K\left(P_{m} ; P_{m-1}\right) \cdots K\left(P_{2} ; P_{1}\right) \\
& \quad \times T\left(P_{1} ; \boldsymbol{r}_{0}\right) S_{f, i}\left(\boldsymbol{r}_{0}, E_{1}, \boldsymbol{\Omega}_{1}\right), \\
& W_{f}^{(1)}\left(P_{m}, \cdots, P_{1}, \boldsymbol{r}_{0}\right)=\frac{\Sigma_{t}\left(P_{\ell}\right)}{\nu \Sigma_{f}\left(P_{\ell}\right)} \frac{\partial}{\partial a}\left(\frac{\nu \Sigma_{f}\left(P_{\ell}\right)}{\Sigma_{t}\left(P_{\ell}\right)}\right) \\
& \quad+\frac{1}{K\left(P_{\ell} ; P_{\ell-1}\right)} \frac{\partial}{\partial a} K\left(P_{\ell} ; P_{\ell-1}\right)+\cdots \\
& \quad+\frac{1}{K\left(P_{2} ; P_{1}\right)} \frac{\partial}{\partial a} K\left(P_{2} ; P_{1}\right)+\frac{1}{T\left(P_{1} ; \boldsymbol{r}_{0}\right)} \frac{\partial}{\partial a} T\left(P_{1} ; \boldsymbol{r}_{0}\right) \\
& \quad+\frac{1}{S_{f, i}\left(\boldsymbol{r}_{0}, E_{1}, \boldsymbol{\Omega}_{1}\right)} \frac{\partial}{\partial a} S_{f, i}\left(\boldsymbol{r}_{0}, E_{1}, \boldsymbol{\Omega}_{1}\right)
\end{aligned}
$$

where $P$ is the six-dimensional vector $(\boldsymbol{r}, E, \boldsymbol{\Omega})$ which represents spatial position $\boldsymbol{r}$, energy $E$, angle $\Omega ; S_{f, i}(P)$ is the fission source; $\Sigma_{t}$ and $\nu \Sigma_{f}$ are the total and production cross sections, respectively; $K$ is the transition kernel which consists of the transport kernel $T$ multiplied by the collision kernel $C$. The transition, transport and collision kernels are defined as follows:

$$
\begin{aligned}
& K\left(P_{\ell} ; P_{\ell-1}\right)=T\left(P_{\ell} ; \boldsymbol{r}_{\ell-1}\right) \\
& \quad \times C\left(\boldsymbol{r}_{\ell-1}, E_{\ell}, \boldsymbol{\Omega}_{\ell} ; E_{\ell-1}, \boldsymbol{\Omega}_{\ell-1}\right), \\
& T\left(\boldsymbol{r}, E, \boldsymbol{\Omega} ; \boldsymbol{r}^{\prime}\right)=\Sigma_{t}(\boldsymbol{r}, E) \\
& \quad \times \exp \left[-\int_{0}^{\left|\boldsymbol{r}-\boldsymbol{r}^{\prime}\right|} \Sigma_{t}\left(\boldsymbol{r}-s \frac{\boldsymbol{r}-\boldsymbol{r}^{\prime}}{\left|\boldsymbol{r}-\boldsymbol{r}^{\prime}\right|}\right) d s\right] \\
& \quad \times \frac{\delta\left(\boldsymbol{\Omega} \cdot \frac{\boldsymbol{r}-\boldsymbol{r}^{\prime}}{\left|\boldsymbol{r}-\boldsymbol{r}^{\prime}\right|}-1\right)}{\left|\boldsymbol{r}-\boldsymbol{r}^{\prime}\right|^{2}}, \\
& C\left(\boldsymbol{r}, E, \boldsymbol{\Omega} ; E^{\prime}, \boldsymbol{\Omega}^{\prime}\right)=\frac{\Sigma_{s}\left(\boldsymbol{r} ; E, \boldsymbol{\Omega} \leftarrow E^{\prime}, \boldsymbol{\Omega}^{\prime}\right)}{\Sigma_{t}\left(\boldsymbol{r}, E^{\prime}\right)} .
\end{aligned}
$$

$W_{f}^{(1)}$ is the weight factor to estimate the first-order differential coefficient.

To express the tally score for the first-order differential coefficient of $k_{i}$ explicitly, Eq. (2) is transformed into the following form:

$$
\begin{aligned}
& \frac{\partial k_{i}}{\partial a}=\frac{1}{\int d P S_{f, i}(P)} \sum_{m=1}^{\infty} \int d P_{m} \cdots \int d P_{1} \int d \boldsymbol{r}_{0} \\
& \times\left[\sum_{\ell=1}^{m} W_{f}^{(1)}\left(P_{\ell}, \cdots, P_{1}, \boldsymbol{r}_{0}\right) \frac{\nu \Sigma_{f}\left(P_{\ell}\right)}{\Sigma_{t}\left(P_{\ell}\right)}\right] \\
& \times H\left(P_{m}, \cdots, P_{1}, \boldsymbol{r}_{0}\right) \\
& H\left(P_{m}, \cdots, P_{1}, \boldsymbol{r}_{0}\right)= \\
& \quad \alpha\left(P_{m}\right) K\left(P_{m} ; P_{m-1}\right) \cdots K\left(P_{2} ; P_{1}\right) \\
& \quad \times T\left(P_{1} ; \boldsymbol{r}_{0}\right) S_{f, i}\left(\boldsymbol{r}_{0}, E_{1}, \boldsymbol{\Omega}_{1}\right)
\end{aligned}
$$

where $\alpha$ is the absorption probability. $H$ denotes a neutron history; it means the existence probability of the neutron. It is unity for analog Monte Carlo and is a particle weight $w$ for non-analog Monte Carlo. Scoring $W_{f}^{(1)} \nu \Sigma_{f} / \Sigma_{t}$ or $W_{f}^{(1)} w \nu \Sigma_{f} / \Sigma_{t}$ estimates the first-order differential coefficient of $k_{i}$ for analog or non-analog Monte Carlo, respectively. Scored values for $W_{f}^{(1)}$ depend on the perturbation parameter; References 6 and 7 can be consulted for concrete examples.
Furthermore, Eq. (8) can be split into the perturbed source and non-perturbed source terms:

$$
\begin{aligned}
\frac{\partial k_{i}}{\partial a}= & \frac{\partial k_{\mathrm{NPS}, i}}{\partial a}+\frac{\partial k_{\mathrm{PS}, i}}{\partial a} \\
\frac{\partial k_{\mathrm{NPS}, i}}{\partial a}= & \frac{1}{\int d P S_{f, i}(P)} \sum_{m=1}^{\infty} \int d P_{m} \cdots \int d P_{1} \int d \boldsymbol{r}_{0} \\
& \times\left[\sum_{\ell=1}^{m} W_{f, \mathrm{NPS}}^{(1)} \frac{\nu \Sigma_{f}\left(P_{\ell}\right)}{\Sigma_{t}\left(P_{\ell}\right)}\right] H \\
\frac{\partial k_{\mathrm{PS}, i}}{\partial a}= & \frac{1}{\int d P S_{f, i}(P)} \sum_{m=1}^{\infty} \int d P_{m} \cdots \int d P_{1} \int d \boldsymbol{r}_{0} \\
& \times\left[\sum_{\ell=1}^{m} W_{f, \mathrm{PS}}^{(1)} \frac{\nu \Sigma_{f}\left(P_{\ell}\right)}{\Sigma_{t}\left(P_{\ell}\right)}\right] H
\end{aligned}
$$

where

$$
\begin{aligned}
& W_{f, \mathrm{NPS}}^{(1)}=\frac{\Sigma_{t}\left(P_{\ell}\right)}{\nu \Sigma_{f}\left(P_{\ell}\right)} \frac{\partial}{\partial a}\left(\frac{\nu \Sigma_{f}\left(P_{\ell}\right)}{\Sigma_{t}\left(P_{\ell}\right)}\right) \\
& \quad+\frac{1}{K\left(P_{\ell} ; P_{\ell-1}\right)} \frac{\partial}{\partial a} K\left(P_{\ell} ; P_{\ell-1}\right)+\cdots \\
& \quad+\frac{1}{K\left(P_{2} ; P_{1}\right)} \frac{\partial}{\partial a} K\left(P_{2} ; P_{1}\right)+\frac{1}{T\left(P_{1} ; \boldsymbol{r}_{0}\right)} \frac{\partial}{\partial a} T\left(P_{1} ; \boldsymbol{r}_{0}\right) \\
& W_{f, \mathrm{PS}}^{(1)}=\frac{1}{S_{f, i}\left(\boldsymbol{r}_{0}, E_{1}, \boldsymbol{\Omega}_{1}\right)} \frac{\partial}{\partial a} S_{f, i}\left(\boldsymbol{r}_{0}, E_{1}, \boldsymbol{\Omega}_{1}\right)
\end{aligned}
$$

The use of Eq. (10) enables us to estimate the PSE and its statistical uncertainty quantitatively.

In a Monte Carlo power iteration, the fission source is normalized as follows:

$$
\begin{aligned}
& S_{f, i}(P)=\frac{1}{k_{i-1}} \int d P^{\prime} \mathcal{K}_{F}\left(P ; P^{\prime}\right) S_{f, i-1}\left(P^{\prime}\right), \\
& \int d P^{\prime} \mathcal{K}_{F}\left(P ; P^{\prime}\right) S_{f, i-1}\left(P^{\prime}\right)= \\
& \sum_{m=0}^{\infty} \int d E_{m} \int d \boldsymbol{\Omega}_{m} \int d P_{m-1} \cdots \int d P_{0} \\
& \quad \times \chi(E, \boldsymbol{\Omega}) F\left(\boldsymbol{r}, E_{m}, \boldsymbol{\Omega}_{m}, P_{m-1}, \cdots, P_{0}\right)
\end{aligned}
$$

Thus the normalization condition is also imposed for the differential coefficient of the fission source. The condition is derived from Eq. (15) as

$$
\begin{aligned}
\frac{\partial}{\partial a} S_{f, i}(P)= & \frac{1}{k_{i-1}}\left\{\int d P^{\prime} \frac{\partial}{\partial a}\left[\mathcal{K}_{F} S_{f, i-1}\left(P^{\prime}\right)\right]\right. \\
& \left.-\frac{1}{k_{i-1}} \frac{\partial k_{i-1}}{\partial a} \int d P^{\prime} \mathcal{K}_{F} S_{f, i-1}\left(P^{\prime}\right)\right\} .
\end{aligned}
$$

This condition must be considered for Eq. (14).

In the present work, the first-order differential coefficient is calculated from the collision estimates in each batch. The final value is obtained by averaging over active batches as follows:

$$
E\left[\frac{\partial k}{\partial a}\right]=\frac{1}{N-N_{\text {skip }}} \sum_{i=N_{\text {skip }}+1}^{N} \frac{\partial k_{i}}{\partial a},
$$


where $E[\partial k / \partial a]$ is the estimated $\partial k / \partial a$ value; $N$ and $N_{\text {skip }}$ are the total and skipped (inactive) batches, respectively. The variance is simply estimated as follows:

$$
\begin{aligned}
& \sigma^{2}\left[\frac{\partial k}{\partial a}\right]=\frac{1}{N-N_{\text {skip }}-1} \\
& \quad \times\left[\frac{1}{N-N_{\text {skip }}} \sum_{i=N_{\text {skip }}+1}^{N}\left(\frac{\partial k_{i}}{\partial a}\right)^{2}-E^{2}\left[\frac{\partial k}{\partial a}\right]\right],
\end{aligned}
$$

where $\sigma[\partial k / \partial a]$ is the standard deviation for the estimated $\partial k / \partial a$ value. The correlation between batches (generations) is not taken into account.

\section{Higher-Order Differential Coefficient}

The formula for the second-order differential coefficient of $k_{i}$ can be derived by differentiating Eq. (2).

$$
\begin{aligned}
\frac{\partial^{2} k_{i}}{\partial a^{2}}= & \frac{1}{\int d P S_{f, i}(P)} \sum_{m=1}^{\infty} \int d P_{m} \cdots \int d P_{1} \int d \boldsymbol{r}_{0} \\
& \times W_{f}^{(2)} F \\
W_{f}^{(2)}= & \frac{\partial W_{f}^{(1)}}{\partial a}+\left(W_{f}^{(1)}\right)^{2}
\end{aligned}
$$

$W_{f}^{(2)}$ is the weight factor to estimate the second-order differential coefficient.

Likewise, we can obtain the formula for the higher-order differential coefficient of $k_{i}$ as follows:

$$
\begin{aligned}
\frac{\partial^{n} k_{i}}{\partial a^{n}}= & \frac{1}{\int d P S_{f, i}(P)} \sum_{m=1}^{\infty} \int d P_{m} \cdots \int d P_{1} \int d \boldsymbol{r}_{0} \\
& \times W_{f}^{(n)} F \\
W_{f}^{(n)}= & \frac{\partial W_{f}^{(n-1)}}{\partial a}+W_{f}^{(n-1)} W_{f}^{(1)}
\end{aligned}
$$

$W_{f}^{(n)}$ can be calculated from $W_{f}^{(1)}$ and its derivatives by using Eq. (23) recursively. The following relation is useful for the recursive calculation:

$$
\frac{\partial^{t} W_{f}^{(n)}}{\partial a^{t}}=\sum_{s=0}^{t} \frac{t !}{s !(t-s) !} \frac{\partial^{s} W_{f}^{(n-1)}}{\partial a^{s}} \frac{\partial^{t-s} W_{f}^{(1)}}{\partial a^{t-s}} .
$$

Equation (22) can be transformed into the tally-score form:

$$
\begin{aligned}
\frac{\partial^{n} k_{i}}{\partial a^{n}}= & \frac{1}{\int d P S_{f, i}(P)} \sum_{m=1}^{\infty} \int d P_{m} \cdots \int d P_{1} \int d \boldsymbol{r}_{0} \\
& \times\left[\sum_{\ell=1}^{m} W_{f}^{(n)} \frac{\nu \Sigma_{f}\left(P_{\ell}\right)}{\Sigma_{t}\left(P_{\ell}\right)}\right] H
\end{aligned}
$$

The higher-order differential coefficients of $k_{i}$ can be estimated by scoring $W_{f}^{(n)} \nu \Sigma_{f} / \Sigma_{t}$ at every collision site. The above procedure has been already proposed by Morillon; ${ }^{3)}$ the detailed algorithm is described in his paper.
To estimate the perturbed source effect explicitly, we split Eq. (25) into two terms:

$$
\begin{aligned}
\frac{\partial^{n} k_{i}}{\partial a^{n}}= & \frac{\partial^{n} k_{\mathrm{NPS}, i}}{\partial a^{n}}+\frac{\partial^{n} k_{\mathrm{PS}, i}}{\partial a^{n}} \\
\frac{\partial^{n} k_{\mathrm{NPS}, i}}{\partial a^{n}}= & \frac{1}{\int d P S_{f, i}(P)} \sum_{m=1}^{\infty} \int d P_{m} \cdots \int d P_{1} \int d \boldsymbol{r}_{0} \\
& \times\left[\sum_{\ell=1}^{m} W_{f, \mathrm{NPS}}^{(n)} \frac{\nu \Sigma_{f}\left(P_{\ell}\right)}{\Sigma_{t}\left(P_{\ell}\right)}\right] H \\
\frac{\partial^{n} k_{\mathrm{PS}, i}}{\partial a^{n}}= & \frac{1}{\int d P S_{f, i}(P)} \sum_{m=1}^{\infty} \int d P_{m} \cdots \int d P_{1} \int d \boldsymbol{r}_{0} \\
& \times\left[\sum_{\ell=1}^{m} W_{f, \mathrm{PS}}^{(n)} \frac{\nu \Sigma_{f}\left(P_{\ell}\right)}{\Sigma_{t}\left(P_{\ell}\right)}\right]
\end{aligned}
$$

where

$$
\begin{aligned}
W_{f, \mathrm{NPS}}^{(n)}= & \frac{\partial W_{f, \mathrm{NPS}}^{(n-1)}}{\partial a}+W_{f, \mathrm{NPS}}^{(n-1)} W_{f, \mathrm{NPS}}^{(1)} \\
W_{f, \mathrm{PS}}^{(n)}= & \frac{\partial W_{f, \mathrm{PS}}^{(n-1)}}{\partial a}+W_{f, \mathrm{NPS}}^{(1)} W_{f, \mathrm{PS}}^{(n-1)} \\
& +\left[W_{f, \mathrm{NPS}}^{(n-1)}+W_{f, \mathrm{PS}}^{(n-1)}\right] W_{f, \mathrm{PS}}^{(1)} .
\end{aligned}
$$

Equations (29) and (30) can be also calculated with Morillon's procedure.

The calculation of Eq.(30) requires the higher-order differential coefficients of the fission source. They are calculated with the following normalization condition in the power iteration:

$$
\begin{aligned}
\frac{\partial^{n}}{\partial a^{n}} & S_{f, i}(P)=\frac{1}{k_{i-1}}\left\{\int d P^{\prime} \frac{\partial^{n}}{\partial a^{n}}\left[\mathcal{K}_{F} S_{f, i-1}\left(P^{\prime}\right)\right]\right. \\
& -\frac{1}{k_{i-1}} \frac{\partial^{n} k_{i-1}}{\partial a^{n}} \int d P^{\prime} \mathcal{K}_{F} S_{f, i-1}\left(P^{\prime}\right) \\
& \left.-\sum_{s=1}^{n-1} \frac{n !}{s !(n-s) !} \frac{\partial^{s}}{\partial a^{s}} S_{f, i-1}\left(P^{\prime}\right) \frac{\partial^{n-s} k_{i-1}}{\partial a^{n-s}}\right\} .
\end{aligned}
$$

Similarly to the first-order differential coefficient, the higher-order differential coefficients are calculated as follows:

$$
E\left[\frac{\partial^{n} k}{\partial a^{n}}\right]=\frac{1}{N-N_{\text {skip }}} \sum_{i=N_{\text {skip }}+1}^{N} \frac{\partial^{n} k_{i}}{\partial a^{n}},
$$

where $E\left[\partial^{n} k / \partial a^{n}\right]$ is the estimated $\partial^{n} k / \partial a^{n}$ value. The variance is estimated as follows:

$$
\begin{aligned}
& \sigma^{2}\left[\frac{\partial^{n} k}{\partial a^{n}}\right]=\frac{1}{N-N_{\text {skip }}-1} \\
& \times\left[\frac{1}{N-N_{\text {skip }}} \sum_{i=N_{\text {skip }}+1}^{N}\left(\frac{\partial^{n} k_{i}}{\partial a^{n}}\right)^{2}-E^{2}\left[\frac{\partial^{n} k}{\partial a^{n}}\right]\right],
\end{aligned}
$$

where $\sigma\left[\partial^{n} k / \partial a^{n}\right]$ is the standard deviation for the estimated $\partial^{n} k / \partial a^{n}$ value. The correlation between batches (generations) is not taken into account. In addition, all the correlations between the differential coefficients are neglected in estimating the reactivity worth; they are caused by using the same random walk to estimate the differential coefficients. 
Table 1 Comparison of the reactivity worth calculated with the DOS method and its reference values for the Godiva assembly (perfectly voided case)

\begin{tabular}{ccc}
\hline & \multicolumn{2}{c}{ Reactivity worth $\left(10^{-3} \Delta k / k k^{\prime}\right)$} \\
\hline Reference & \multicolumn{2}{c}{$-2.883(0.042)^{*}$} \\
\hline Taylor series & Without PSE & With PSE \\
\hline up to 1st-order & $-1.291(0.020)$ & $-3.044(0.054)$ \\
up to 2nd-order & $-1.352(0.023)$ & $-2.803(0.068)$ \\
up to 3rd-order & $-1.346(0.023)$ & $-2.861(0.078)$ \\
up to 4th-order & $-1.350(0.023)$ & $-2.850(0.085)$ \\
\hline * The value in the parenthesis stands for one standard deviation of \\
the statistical uncertainty.
\end{tabular}

\section{Benchmark Calculation}

Benchmark calculations are performed for Godiva, Jezebel and TCA assemblies. The first two assemblies are a fast uranium system and a fast plutonium system, respectively. The TCA assembly is a thermal uranium system. JENDL-3.3 ${ }^{8)}$ has been used for all calculations.

\section{Godiva}

The benchmark model specified in HEU-MET-FAST-001 of the ICSBEP handbook ${ }^{9)}$ is employed; the geometry of the assembly is a bare uranium sphere of a radius of $8.741 \mathrm{~cm}$ and the composition is $93.71 \mathrm{wt} \% \mathrm{U}-235,5.27 \mathrm{wt} \% \mathrm{U}-238$ and $1.02 \mathrm{wt} \% \mathrm{U}-234$. The reactivity worth is calculated for the case where the central region of radius less than $1 \mathrm{~cm}$ is voided. This is a calculation benchmark since the perturbation is fictitiously introduced: such an experiment has not been performed.

The reference values are obtained with two independent Monte Carlo runs performed for 40,000 active and 100 inactive batches with 10,000 histories per batch. A single eigenvalue calculation is, on the other hand, performed for 4,000 active and 100 inactive batches with 10,000 histories per batch to obtain the reactivity worth with the DOS method.

Table 1 shows the comparison of the change in reactivity worth calculated with the DOS method and its reference values for the Godiva assembly. The significant discrepancy can be observed unless the PSE is not taken into account. Comparing the calculated values including the PSE, the first-order approximation yields a slight overestimation of the negative reactivity worth; the values agree within two standard deviations though they do not agree within one standard deviation. The second-order perturbed source effect improves the calculated result; the values agree within one standard deviation. The higher-order approximation also gives a very good agreement with the reference value; the difference from the reference value is less than $1 \%$.

To examine the applicability of the first- and second-order approximation against the void fraction, we plot the change in reactivity worth as a function of the fractional decrease in density. Figure 1 shows the comparison of the change in reactivity worth for the first- and second-order approximations. The

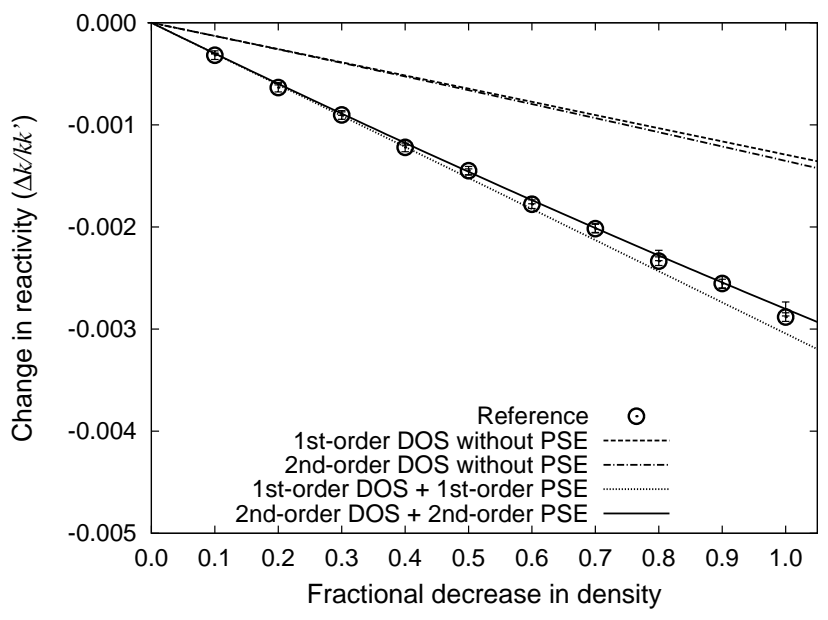

Fig. 1 Comparison of the change in reactivity worth calculated with the differential operator sampling method and its reference values for the Godiva assembly

first-order approximation is applicable up to about $50 \%$ decrease in density but the discrepancy becomes larger for more than $50 \%$ decrease. The second-order approximation is, on the other hand, applicable for the perfectly voided case $(100 \%$ decrease in density).

\section{Jezebel}

We perform a benchmark calculation for a $\mathrm{Pu}-238$ replacement measurement using Jezebel. This experiment has been reported as SPEC-MET-FAST-002 in the ICSBEP handbook. The benchmark geometry of the assembly is a bare plutonium sphere of a radius of $6.6595 \mathrm{~cm}$ and the composition is 76.4 at $\% \mathrm{Pu}-239,20.1$ at $\% \mathrm{Pu}-240,3.1$ at $\% \mathrm{Pu}-241$ and 0.4 at $\% \mathrm{Pu}-$ 242. The $\mathrm{Pu}-238$ sample of a radius of $0.6439 \mathrm{~cm}$ is placed at the center of the Jezebel assembly. The reactivity worth is calculated as the change in reactivity between the $\mathrm{Pu}-238$ sample and a void.

The reference values are obtained with two independent Monte Carlo runs performed for 160,000 active and 100 inactive batches with 10,000 histories per batch. A single eigenvalue calculation is, on the other hand, performed for 10,000 active and 100 inactive batches with 10,000 histories per batch to obtain the reactivity worth with the DOS method.

Table 2 shows the comparison of the change in reactivity worth calculated with the DOS method and its reference values for the Jezebel assembly. Almost the same trend as for Godiva is observed for the Jezebel case. The PSE must be calculated and the second- or higher order approximation gives a very good result; the values agree within one standard deviation and the difference is less than $1 \%$.

According to the ICSBEP handbook, the experimental value is $1.461( \pm 0.109) \times 10^{-3} \Delta k / k k^{\prime}$. The $\beta_{\text {eff }}$ value of $0.00204 \pm 0.00011$ is used for the unit conversion. All the calculated data agree with the experimental value within the experimental uncertainty. We do not perform further investigation for validation of nuclear data in the present work. However, the reactivity worth calculation with the DOS method can be an applicable tool for it. 
Table 2 Comparison of the reactivity worth calculated with the DOS method and its reference values for the Jezebel assembly (perfectly voided case)

\begin{tabular}{ccc}
\hline & \multicolumn{2}{c}{ Reactivity worth $\left(10^{-3} \Delta k / k k^{\prime}\right)$} \\
\hline Reference & \multicolumn{2}{c}{$-1.392(0.021)^{*}$} \\
\hline Taylor series & Without PSE & With PSE \\
\hline up to 1st-order & $-0.742(0.007)$ & $-1.470(0.020)$ \\
up to 2nd-order & $-0.767(0.007)$ & $-1.382(0.022)$ \\
up to 3rd-order & $-0.767(0.007)$ & $-1.391(0.022)$ \\
up to 4th-order & $-0.767(0.007)$ & $-1.391(0.022)$ \\
\hline * The value in the parenthesis stands for one standard deviation of \\
the statistical uncertainty.
\end{tabular}

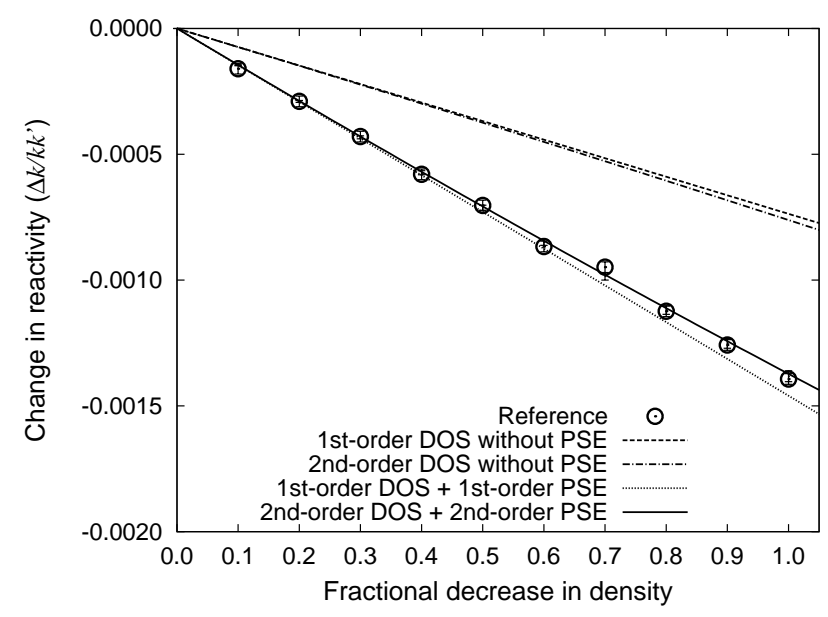

Fig. 2 Comparison of the change in reactivity worth calculated with the differential operator sampling method and its reference values for the Jezebel assembly

Figure 2 shows the comparison of the change in reactivity worth for the first- and second-order approximations. The second-order approximation is applicable for the perfectly voided case ( $100 \%$ decrease in density) but the first-order approximation tends to overestimate the reference values of the negative reactivity worth slightly as the decrease in density becomes larger.

\section{TCA}

Measurements of Np-237 sample reactivity worth ${ }^{10)}$ have been performed with the Tank-Type Critical Assembly (TCA), which is a light-water moderated and reflected $2.6 \mathrm{wt} \%$ enriched $\mathrm{UO}_{2}$ lattice. ${ }^{5)}$ The sample worth was measured for cores with various water-to-fuel volume ratios. We perform benchmark calculations for a core of the water-to-fuel volume ratio of 3.0 because the measured reactivity worth is more than 20 cent $\left(>0.0015 \Delta k / k k^{\prime}\right)$. Figure 3 shows the calculation geometry for the benchmark. The experimental channel and the sample are explicitly modeled in the geometry. References 5 and 10 can be consulted for detailed information such as dimensions, composition, etc. about TCA and the sample, respectively.
Table 3 Comparison of the Np-237 sample reactivity worth calculated with the DOS method and its reference values for TCA (perfectly voided case)

\begin{tabular}{ccc}
\hline & \multicolumn{2}{c}{ Reactivity worth $\left(10^{-3} \Delta k / k k^{\prime}\right)$} \\
\hline Reference & \multicolumn{2}{c}{$1.754(0.014)^{*}$} \\
\hline Taylor series & Without PSE & With PSE \\
\hline up to 1st-order & $0.511(0.004)$ & $0.793(0.013)$ \\
up to 2nd-order & $0.754(0.005)$ & $1.194(0.017)$ \\
up to 3rd-order & $0.872(0.006)$ & $1.385(0.021)$ \\
up to 4th-order & $0.932(0.007)$ & $1.494(0.025)$ \\
up to 5th-order & $0.961(0.008)$ & $1.552(0.029)$ \\
up to 6th-order & $0.975(0.009)$ & $1.563(0.033)$ \\
up to 7th-order & $0.980(0.009)$ & $1.579(0.038)$ \\
up to 8th-order & $0.982(0.010)$ & $1.562(0.042)$ \\
\hline
\end{tabular}

* The value in the parenthesis stands for one standard deviation of the statistical uncertainty.

The reference values are obtained with two independent Monte Carlo runs performed for 100,000 active and 100 inactive batches with 64,000 histories per batch. A single eigenvalue calculation is, on the other hand, performed for 8,000 active and 100 inactive batches with 64,000 histories per batch to obtain the reactivity worth with the DOS method.

Table 3 shows the comparison of the change in reactivity worth calculated with the DOS method and its reference values for the TCA assembly. The Taylor series terms are calculated up to eighth order for this problem. The PSE terms are also calculated up to eighth order. However, the sixth and higher-order PSE terms have not been confidently estimated because the estimates of the differential coefficients fluctuate significantly as the number of the propagation batches increases and the relative statistical errors of the differential coefficients are larger than 100\%. The second-order approximation underestimates the reference result by $\sim 30 \%$ even if the PSE is taken into account although the approximation gives very good results for the Godiva and Jezebel cases. Consideration of the higher-order PSE improves the calculated result but the fifth-order approximation still underestimates the reference result by $\sim 11 \%$. Therefore, the higher-order Taylor series must be taken into account and the sixth- and higherorder PSE terms must be estimated accurately for the TCA case.

To investigate the applicability of the DOS method, the change in reactivity worth is plotted as a function of the fractional decrease in density. Figure 4 shows the comparison between Taylor approximations and the reference solution for the case where the PSE is not taken into account. Though the reactivity change can be confidently estimated up to the higher order in this case, the calculated results are significantly underestimated in all the range of the fractional decrease. Figure 5 shows, on the other hand, for the case where the PSE is taken into account. The third- or higher-order approximation gives a very good result until the density decrease of $50 \%$. However, the discrepancy becomes large even for the fifth- 


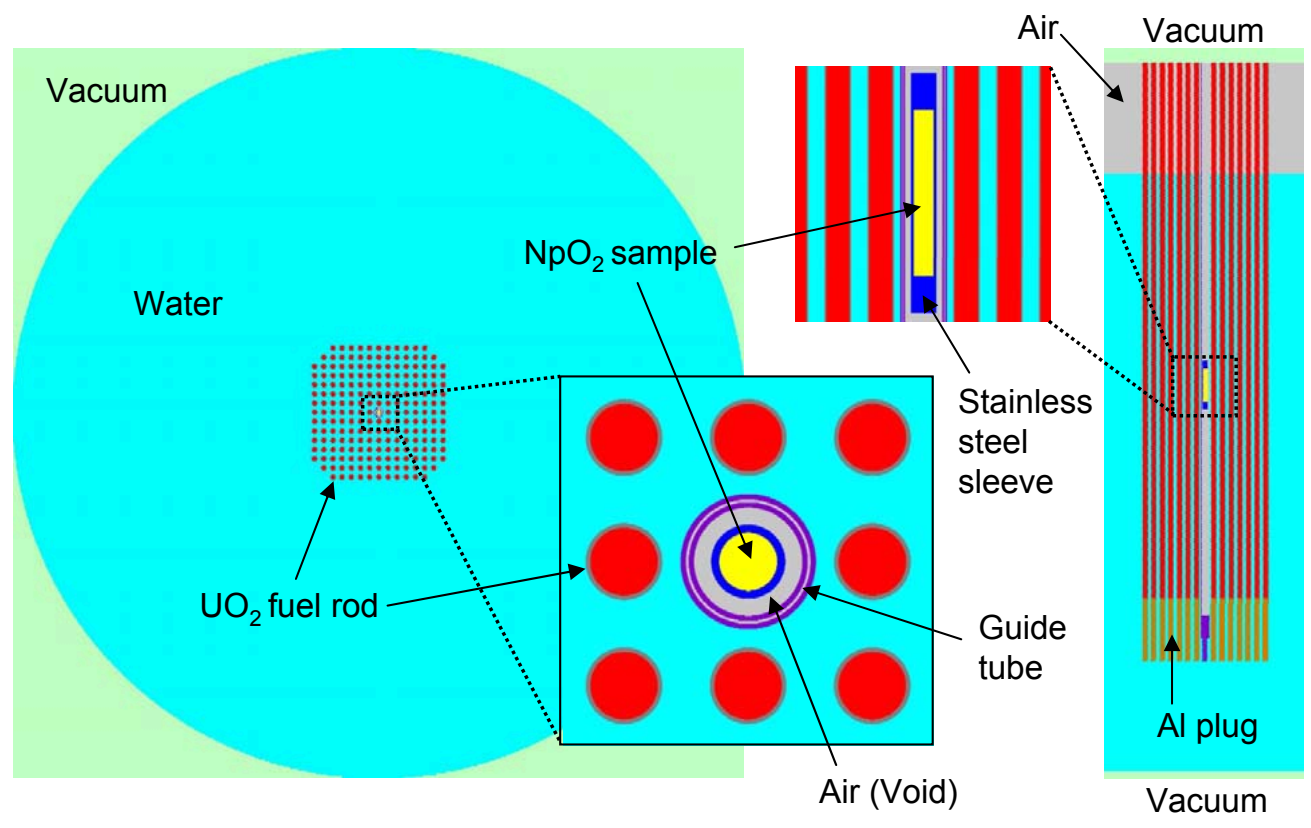

Fig. 3 Calculation geometry for the TCA assembly

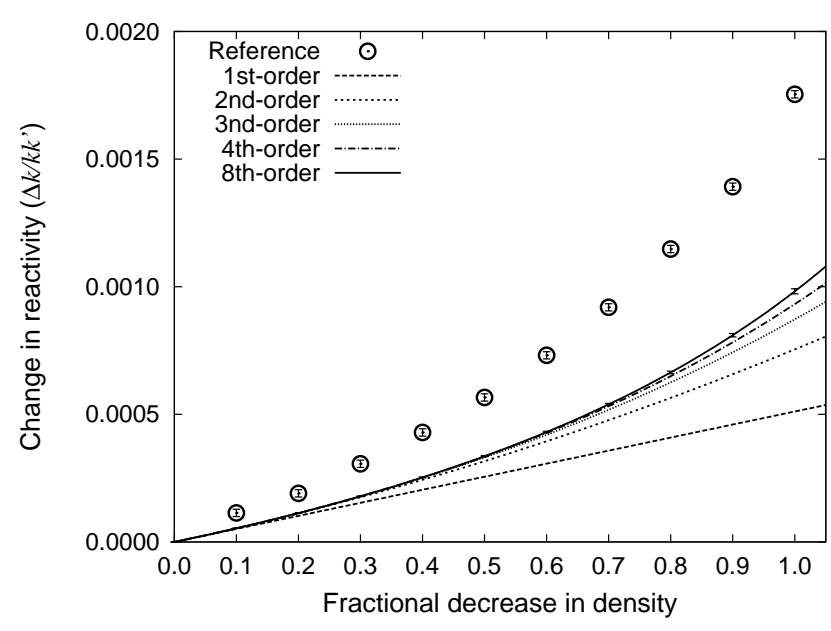

Fig. 4 Comparison of the change in reactivity worth calculated with the differential operator sampling method and its reference values for the TCA assembly (no PSE included)

order approximation as the density of the sample decreases. These results suggest that more higher-order terms must be considered.

\section{Alternative Approaches}

In the previous section, it has been found that the accurate estimation of the sample reactivity worth with the ordinary Taylor series approach is difficult for the TCA case. To mitigate this difficulty, three alternative approaches are examined: the Padé approximation, ${ }^{11)}$ midpoint method and the interpoint method. The Padé approximation is expected to improve the estimation of higher-order effects. The midpoint and interpoint methods are expected to improve the accuracy of the

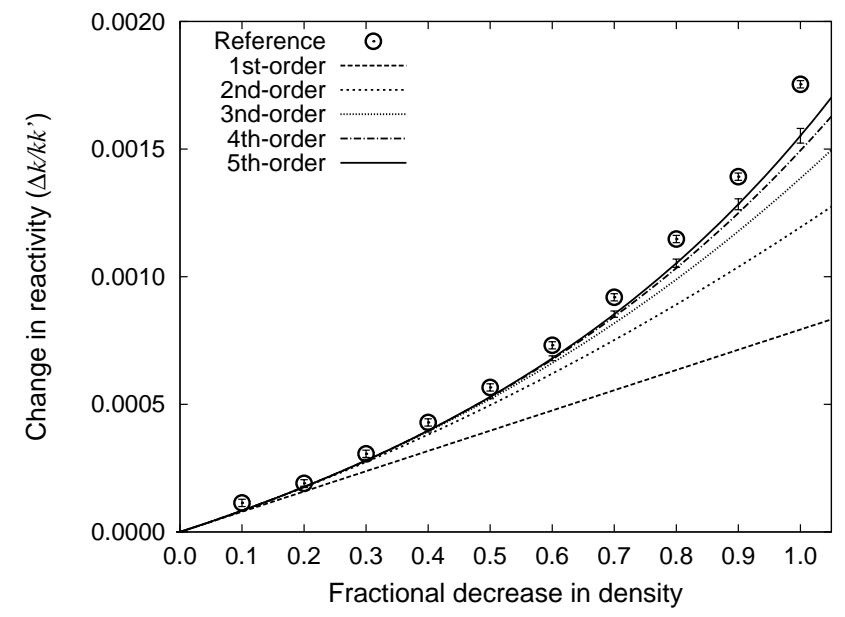

Fig. 5 Comparison of the change in reactivity worth calculated with the differential operator sampling method and its reference values for the TCA assembly (PSE included)

calculated sample reactivity worth by reducing its magnitude and the density change.

\section{Padé approximation}

Suppose that the effective multiplication factor $k$ is a function of the variation of a perturbation parameter $x=\Delta a$. Then the Taylor series of $k(x)$ at $x=0$ can be expressed as follows:

$$
\begin{aligned}
k(x)= & k(0)+k^{\prime}(0) x+\frac{1}{2} k^{\prime \prime}(0) x^{2}+\cdots \\
& +\frac{1}{n !} k^{(n)} x^{n}+\cdots
\end{aligned}
$$




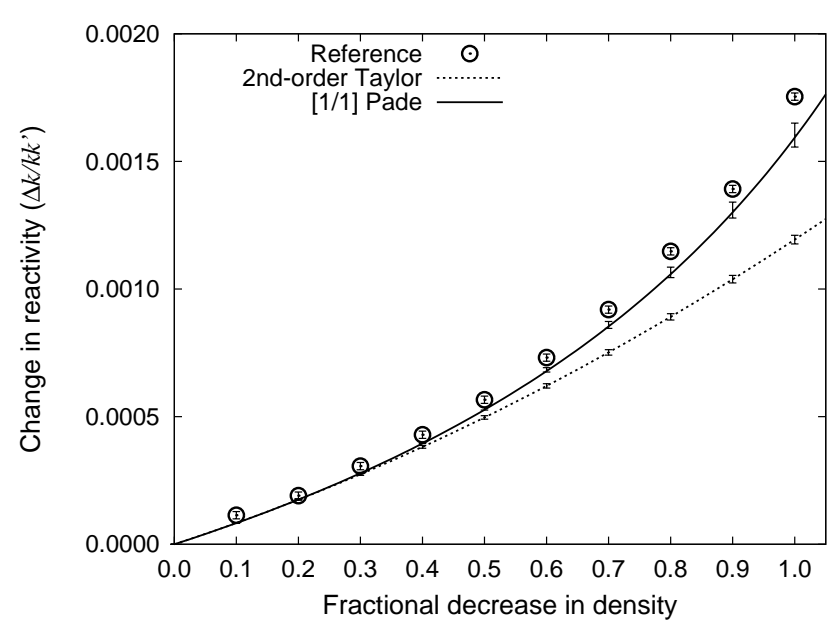

Fig. 6 Comparison of the change in reactivity worth calculated with the second-order Taylor series and the [1/1] Padé approximant for the TCA assembly

With the Padé approximation $k(x)$ can be written as a rational function:

$$
R[L / M](x)=\frac{p_{0}+p_{1} x+p_{2} x^{2}+\cdots+p_{L} x^{L}}{1+q_{1} x+q_{2} x^{2}+\cdots+q_{M} x^{M}} .
$$

To transform Eq. (34) into the rational form, the following conditions must be satisfied:

$$
\begin{aligned}
R[L / M](0) & =k(0), \\
\left.\frac{d^{k}}{d x^{k}} R[L / M](x)\right|_{x=0} & =\left.\frac{d^{k}}{d x^{k}} k(x)\right|_{x=0} \\
& \text { for } k=1,2, \cdots, L+M .
\end{aligned}
$$

The second-order Taylor series can be written with the [1/1] Padé approximant:

$$
k(0)+k^{\prime}(0) x+\frac{1}{2} k^{\prime \prime}(0) x^{2} \approx \frac{p_{0}+p_{1} x}{1+q_{1} x},
$$

where the coefficients $p_{0}, p_{1}$ and $q_{1}$ can be obtained from Eqs. (36) and (37). Thus the change in $k$ is expressed as follows:

$$
\begin{aligned}
\Delta k & =R[1 / 1](x)-R[1 / 1](0) \\
& =\frac{\left(p_{1}-p_{0} q_{1}\right) x}{1+q_{1} x}=\frac{k^{\prime}(0) \Delta a}{1-\frac{k^{\prime \prime}(0)}{2 k^{\prime}(0)} \Delta a} .
\end{aligned}
$$

Likewise, the fourth-order Taylor series can be written with the [2/2] Padé approximant:

$$
\begin{aligned}
k(0)+k^{\prime}(0) x+\frac{1}{2} k^{\prime \prime}(0) x^{2}+ & \frac{1}{3 !} k^{\prime \prime \prime}(0) x^{3}+\frac{1}{4 !} k^{(4)}(0) x^{4} \\
& \approx \frac{p_{0}+p_{1} x+p_{2} x^{2}}{1+q_{1} x+q_{2} x^{2}}
\end{aligned}
$$

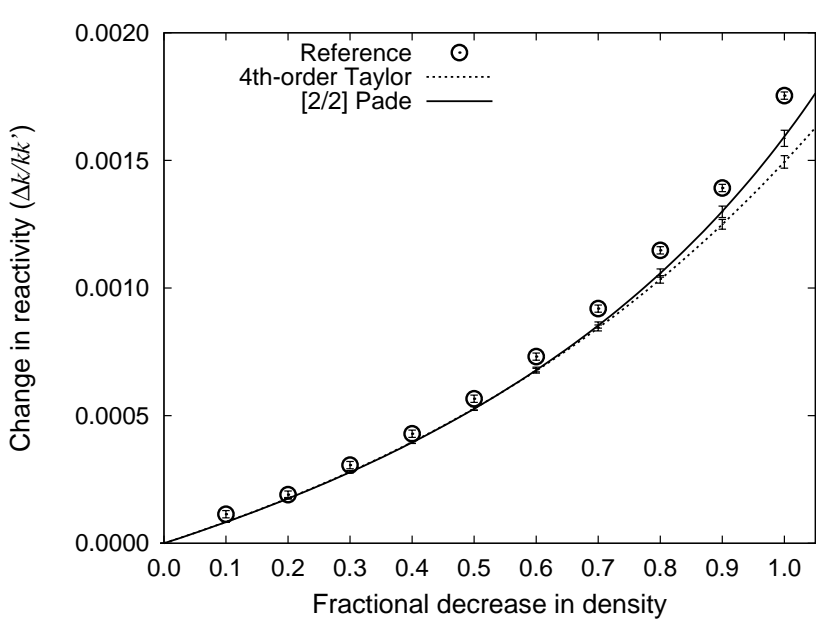

Fig. 7 Comparison of the change in reactivity worth calculated with the fourth-order Taylor series and the [2/2] Padé approximant for the TCA assembly

Table 4 Comparison of the Np-237 sample reactivity worth calculated with the Padé approximation and its reference value for TCA (perfectly voided case)

\begin{tabular}{cc}
\hline & Reactivity worth $\left(10^{-3} \Delta k / k k^{\prime}\right)$ \\
\hline Reference & $1.754(0.014)^{*}$ \\
\hline 2nd-order Taylor series & $1.194(0.017)^{* *}$ \\
4th-order Taylor series & $1.494(0.025)$ \\
\hline$[1 / 1]$ Padé & $1.603(0.047)$ \\
{$[2 / 2]$ Padé } & $1.587(0.032)$ \\
\hline
\end{tabular}

* The value in the parenthesis stands for one standard deviation of the statistical uncertainty.

** All the calculated results include the PSE.

The change in $k$ is expressed as follows:

$$
\begin{aligned}
\Delta k & =R[2 / 2](x)-R[2 / 2](0) \\
& =\frac{c_{1} \Delta a+c_{2}(\Delta a)^{2}}{1+q_{1} \Delta a+q_{2}(\Delta a)^{2}}, \\
c_{1} & =p_{1}-p_{0} q_{1}=k^{\prime}(0), \\
c_{2} & =p_{2}-p_{0} q_{2}=q_{1} k^{\prime}(0)+\frac{1}{2} k^{\prime \prime}(0), \\
q_{1} & =\frac{2 k^{\prime \prime \prime}(0) k^{\prime}(0)-6 k^{(4)}(0) k^{\prime}(0)}{4 k^{\prime \prime \prime}(0) k^{\prime}(0)-6\left(k^{\prime \prime}(0)\right)^{2}}, \\
q_{2} & =\frac{4\left(k^{\prime \prime \prime}(0)\right)^{2}-3 k^{(4)}(0) k^{\prime \prime}(0)}{36\left(k^{\prime \prime}(0)\right)^{2}-24 k^{\prime}(0) k^{\prime \prime \prime}(0)} .
\end{aligned}
$$

Figures 6 and 7 show the comparison of the change in the reactivity worth calculated with the second-order Taylor series and the [1/1] Padé approximant, and with the fourthorder Taylor series and the [2/2] Padé approximant, respectively. The Padé approximation gives better results than the Taylor series approximations. In particular, the [1/1] Padé approximation outperforms the second-order Taylor series approximation for the large fractional decrease in density even though the same differential coefficients up to the second or- 
Table 5 Comparison of the Np-237 sample reactivity worth calculated by using the midpoint and interpoint methods with the Taylor series approximation and its reference value for TCA (perfectly voided case)

\begin{tabular}{lcc}
\hline & \multicolumn{2}{c}{ Reactivity worth $\left(10^{-3} \Delta k / k k^{\prime}\right)$} \\
\hline Reference & \multicolumn{2}{c}{$1.754(0.014)^{*}$} \\
\hline & Midpoint & Interpoint \\
\hline up to 1st-order & $1.415(0.013)^{* *}$ & $1.793(0.018)$ \\
up to 2nd-order & - & $1.312(0.026)$ \\
up to 3rd-order & $1.577(0.015)$ & $1.771(0.036)$ \\
up to 4th-order & - & $1.531(0.050)$ \\
up to 5th-order & $1.611(0.018)$ & $1.707(0.071)$ \\
up to 6th-order & - & $1.593(0.107)$ \\
up to 7th-order & $1.640(0.021)$ & $1.548(0.156)$ \\
up to 8th-order & - & $1.423(0.174)$ \\
\hline * The value in the parenthesis stands for one standard deviation of \\
the statistical uncertainty. \\
$* *$ All the calculated results include the PSE.
\end{tabular}

der are used. In addition, the low-order Padé approximation gives similar results as the higher-order Taylor series approximation. However, the discrepancies of $\sim 10 \%$ can be still observed for the perfectly voided case calculated with the Padé approximation as shown in Table 4.

\section{Midpoint and Interpoint Methods}

The interpoint method calculates the sample reactivity worth at the interpoint between the cases where the sample is inserted and is perfectly withdrawn. Namely, the positiveand negative-side reactivity worths from the interpoint (reference point) are calculated at the same time. The worths can be expressed as follows:

$$
\begin{aligned}
\Delta k^{+} & =k^{\prime}(0) \Delta a^{+}+\frac{1}{2} k^{\prime \prime}(0)\left(\Delta a^{+}\right)^{2} \\
& +\frac{1}{3 !} k^{\prime \prime \prime}(0)\left(\Delta a^{+}\right)^{3}+\cdots, \\
\Delta k^{-} & =k^{\prime}(0)\left(-\Delta a^{-}\right)+\frac{1}{2} k^{\prime \prime}(0)\left(-\Delta a^{-}\right)^{2} \\
& +\frac{1}{3 !} k^{\prime \prime \prime}(0)\left(-\Delta a^{-}\right)^{3}+\cdots .
\end{aligned}
$$

The total reactivity worth is then calculated as

$$
\Delta k=\Delta k^{+}-\Delta k^{-} .
$$

If we select such that $\Delta a=\Delta a^{+}=\Delta a^{-}$, Eq. (48) is simplified as follows:

$$
\Delta k=2 k^{\prime}(0) \Delta a+\frac{2}{3 !} k^{\prime \prime \prime}(0)(\Delta a)^{3}+\cdots
$$

This is the midpoint method. It has an advantage that the midpoint method with the odd-order Taylor series approximation yields the same result as that with one more higher-order Taylor series.

For the interpoint method, the reference point (interpoint) is set to the system with the Np-237 sample voided by $66.7 \%$
Table 6 Positive- and negative-side reactivity worths calculated by using the interpoint method with the Taylor series approximation and their reference values

\begin{tabular}{ccc}
\hline & \multicolumn{2}{c}{ Reactivity worth $\left(10^{-3} \Delta k / k k^{\prime}\right)$} \\
\hline & $\begin{array}{c}\text { Positive side } \\
+200 \% \text { increase }\end{array}$ & $\begin{array}{c}\text { Negative side } \\
-100 \% \text { decrease }\end{array}$ \\
\hline Reference & $-0.843(0.014)^{*}$ & $0.911(0.014)$ \\
\hline \multicolumn{3}{c}{ Interpoint } \\
\hline up to 1st-order & $-1.195(0.016)^{* *}$ & $0.598(0.008)$ \\
up to 2nd-order & $-0.554(0.024)$ & $0.758(0.009)$ \\
up to 3rd-order & $-0.962(0.034)$ & $0.809(0.010)$ \\
up to 4th-order & $-0.706(0.049)$ & $0.825(0.010)$ \\
up to 5th-order & $-0.876(0.070)$ & $0.830(0.010)$ \\
up to 6th-order & $-0.761(0.106)$ & $0.832(0.010)$ \\
up to 7th-order & $-0.716(0.156)$ & $0.832(0.010)$ \\
up to 8th-order & $-0.592(0.174)$ & $0.831(0.010)$ \\
\hline
\end{tabular}

* The value in the parenthesis stands for one standard deviation of the statistical uncertainty.

** All the calculated results include the PSE.

so that the positive- and negative reactivity worths are roughly the same. Then the positive reactivity worth is calculated for the $200 \%$ increase in the sample density and the negative one is for the $100 \%$ decrease in the density. Table $\mathbf{5}$ shows the comparison of the $\mathrm{Np}-237$ sample reactivity worth calculated by using the midpoint and interpoint methods with the Taylor series approximation. The results for the midpoint method becomes better as the order of the Taylor series becomes higher. However, there still exists the discrepancy of $\sim 7 \%$ even for the seventh-order result. The interpoint method, on the other hand, yields the fluctuated results with the order. This fluctuation is caused by the positive-side reactivity worth as seen in Table 6. Since the positive-side reactivity worth is calculated for more than $100 \%$ change in the density, the higher-order terms of the Taylor series becomes large.

To circumvent the fluctuation of the Taylor series approximation, the Padé approximation is applied for the interpoint method. Table 7 shows the Np-237 sample reactivity worth calculated by using the interpoint method with the Padé approximation. The Padé approximation yields the relatively stable results for the positive-side reactivity worth. However, the interpoint method with Padé approximation still underestimates the reference result for the total reactivity worth; it gives similar results as the ordinary Taylor series approximation.

\section{Concluding Remarks}

The applicability of the Taylor series approach with the DOS method has been examined for the calculation of sample reactivity worth. The alternative approaches such as the Padé approximation, the midpoint and interpoint methods are also examined. The higher-order DOS method including the PSE has been formulated. The methodology has been implemented into the MVP code to perform benchmark calculations. The following conclusions have been obtained. 
Table 7 Positive- and negative-side reactivity worths calculated by using the interpoint method with the Padé approximation and their reference value

\begin{tabular}{cccc}
\hline \multicolumn{4}{c}{ Reactivity worth $\left(10^{-3} \Delta k / k k^{\prime}\right)$} \\
\hline $\begin{array}{c}\text { Positive } \\
(+200 \%)\end{array}$ & $\begin{array}{c}\text { Negative } \\
(-100 \%)\end{array}$ & Total \\
\hline Reference & $-0.843(0.014)^{*}$ & $0.911(0.014)$ & $1.754(0.014)$ \\
\hline$[1 / 1]$ Padé & $-0.783(0.008)^{* *}$ & $0.821(0.009)$ & $1.602(0.012)$ \\
\hline$[2 / 2]$ Padé & $-0.810(0.037)$ & $0.837(0.011)$ & $1.644(0.038)$ \\
\hline
\end{tabular}

* The value in the parenthesis stands for one standard deviation of the statistical uncertainty.

** All the calculated results include the PSE.

- The second-order Taylor approximation gives very good results for the sample reactivity worth calculation of the Godiva and Jezebel assemblies; the calculated values agree within one standard deviation.

- The higher-order Taylor terms must be calculated for the Np-237 sample reactivity worth calculation of the TCA assembly. It is, however, difficult to estimate the higher-order PSE confidently because of the large statistical fluctuation.

- The PSE has a significant contribution for all the cases: $\sim 53 \%, \sim 49 \%$ and $\sim 44 \%$ for Godiva, Jezebel and TCA, respectively. It must be thus estimated for the accurate calculation of the reactivity worth.

- The Padé approximation with the low-order differential coefficients yields similar reactivity worths as the higherorder Taylor series approximation. However, there still exists a discrepancy between the calculated result with the Padé approximation and the reference result for the $\mathrm{Np}-237$ sample reactivity worth at TCA.

- Both the midpoint method with the Taylor series approximation and interpoint methods with the Padé approximation give similar results as the ordinary Taylor series approximation for $\mathrm{Np}-237$ sample reactivity worth at TCA; no improvement can be observed.

- The Padé approximation gives better results than the Taylor series approximation for a change larger than $100 \%$.

In the present work, a case has been shown where it is difficult to estimate the sample reactivity worth with the Taylor series approach and the DOS method. The estimation of the higherorder effect gives a better approximate result even for such a case. Further investigation is, however, required for the accurate estimation of sample reactivity worth. Further research for the area of applicability is also required.

\section{Acknowledgments}

The authors are grateful to Professor Ken Nakajima of Kyoto University for his support on the present study and to Dr. Takeshi Sakurai of Japan Atomic Energy Agency for providing useful information on the sample worth measurements at TCA.

\section{References}

1) Y. Nagaya, T. Mori, "Impact of Perturbed Fission Source on the Effective Multiplication Factor in Monte Carlo Perturbation Calculations," J. Nucl. Sci. Technol., 42[5], 428-441 (2005).

2) K. F. Raskash, "An Improvement of the Monte Carlo Generalized Differential Operator Method by Taking into Account First- and Second-Order Perturbations of Fission Source," Nucl. Sci. Eng., 162, 158-166 (2009).

3) B. Morillon, "On the use of Monte Carlo perturbation in neutron transport problems," Ann. Nucl. Energy, 25, 1095-1117 (1998).

4) Y. Nagaya, K. Okumura, T. Mori, M. Nakagawa, $M V P / G M V P$ II: General Purpose Monte Carlo Codes for Neutron and Photon Transport Calculations Based on Continuous Energy and Multigroup Methods, JAERI 1348, Japan Atomic Energy Research Institute (JAERI) (2005).

5) H. Tsuruta et al., Critical Sizes of Light-Water Moderated $\mathrm{UO}_{2}$ and $\mathrm{PuO}_{2}-\mathrm{UO}_{2}$ Lattices, JAERI 1254, Japan Atomic Energy Research Institute (JAERI) (1978).

6) H. Rief, "Generalized Monte Carlo perturbation algorithms for correlated sampling and a second-order Taylor series approach," Ann. Nucl. Energy, 11, 455-476 (1984).

7) D. E. Peplow, K. Verghese "Differential Sampling for the Monte Carlo Practitioner," Prog. Nucl. Energy, 36, 39-75 (2000).

8) K. Shibata et al., "Japanese Evaluated Nuclear Data Library Version 3 Revision-3: JENDL-3.3," J. Nucl. Sci. Technol., 39[11], 1125-1136 (2002).

9) OECD/NEA Nuclear Science Committee, "International Handbook of Evaluated Criticality Safety Benchmark Experiments," NEA/NSC/DOC(95)03, September 2008 Edition (2008), [CDROM].

10) T. Sakurai et al., "Measurement and Analysis of Reactivity Worth of ${ }^{237} \mathrm{~Np}$ Sample in Cores of TCA and FCA," J. Nucl. Sci. Technol., 46[6], 624-640 (2009).

11) G. A. Baker, Jr., Essentials of Padé Approximants, Academic Press, New York (1975). 